\title{
Slow Waves in Fractures Filled with Viscous Fluid
}

\section{Valeri A. Korneev (Lawrence Berkeley National Laboratory Berkeley, CA}

94720)

\begin{abstract}
Stoneley guided waves in a fluid-filled fracture generally have larger amplitudes than other waves, and therefore, their properties need to be incorporated in more realistic models. In this study, a fracture is modeled as an infinite layer of viscous fluid bounded by two elastic half-spaces with identical parameters. For small fracture thickness, I obtain a simple dispersion equation for wave-propagation velocity. This velocity is much smaller than the velocity of a fluid wave in a Biot-type solution, in which fracture walls are assumed to be rigid. At seismic prospecting frequencies and realistic fracture thicknesses, the Stoneley guided wave has wavelengths on the order of several meters and an attenuation Q factor exceeding 10, which indicates the possibility of resonance excitation in fluid-bearing rocks. The velocity and attenuation of Stoneley guided waves are distinctly different at low frequencies for water and oil. The predominant role of fractures in fluid flow at field scales is supported by permeability data showing an increase of several orders of magnitude when compared to values obtained at laboratory scales. These data suggest that Stoneley guided waves should be taken into account in theories describing seismic wave propagation in fluidsaturated rocks.
\end{abstract}




\section{INTRODUCTION}

Stoneley guided waves in fractures are described by Ferazzini and Aki (1987), who use them to explain low-frequency tremors observed before volcanic eruptions. Earlier, Chouet (1986) suggested that such tremors are caused by resonances in molten lava during fracture opening. Because of the large $(0.5-1.0 \mathrm{~m})$ fracture thickness in those earlier investigations, the fluid viscosity was not considered. Such a slow fluid wave is a Stoneley type wave, one that propagates in a waveguide formed by a fluid layerbounded by elastic walls. Krauklis (1962) first described such a wave, but no application was mentioned. Ferazzini and Aki (1987) rederived the slow Stoneley wave as a fundamental symmetric mode that propagates along the fracture with a velocity that approaches zero at low frequency. They stopped short of deriving the low-frequency asymptote for a phase velocity of this wave (see their equation 16), which can be obtained in the form

$$
V_{f 0}=\left(\frac{\omega h \mu}{\rho_{f}}\left(1-\gamma^{2}\right)\right)^{\frac{1}{3}},
$$

where frequency is $\omega$, fracture thickness is $h$, fluid density is $\rho_{f}$, shear modulus of the elastic walls is $\mu$, and the elastic velocities ratio is $\gamma=\mathrm{v}_{\mathrm{s}} / \mathrm{v}_{\mathrm{p}}$.

Fluid-filled fracture waves also have been studied both numerically and in a laboratory (Paillet and White, 1982; Groenenboom and Falk, 2000; Groenenboom and van Dam, 2000; Groenenboom and Fokkema, 1998), for the purpose of monitoring hydrofracture processes, including the effect of diffraction at the tip of the fracture. Those studies confirmed the existence of a slow fluid wave in such models and demonstrated its predominantly high amplitude (Groenenboom and Fokkema, 1998). The dispersive wave represented by equation 
1 has a variety of different names (e.g., slow fluid wave, fluid guided wave, Stoneley guided wave, first symmetric fluid mode), all of which have the same meaning.

Molotkov and Bakulin (1998) showed that for infinite fracture models, a stack of fractures filled with nonviscous fluid has a solution in the form of the second Biot's wave propagating in a transverse isotropic medium with frequency-independent velocity. Goloshubin et al. (1993, 1994) found that the slow Stoneley-type wave can also propagate in such medium when it is sandwiched between two elastic half-spaces. An unbounded stack of fractures containing viscous fluid is capable of carrying frequency-dependent slow Biot- type waves (Gurevich, 2002; Schoenberg, 1984, 1983). It is yet unclear if such a model could also carry a Stoneley-type wave, which has a lower velocity.

Slow fluid waves are essential for generating tube-wave reflections from intersecting fractures (Hornby et al., 1989; Kostek et al., 1998 a, b). The high amplitudes of such waves make the solution of relevant problems rather simple, because we can ignore most other types of waves without compromising the result. Comparisons with exact solutions (Kostek et al., 1998a) showed that considering only fluid waves in seemingly difficult problems is well justified. The energy-trapping ability of such waves illustrates the distinctive feature of fluidfilled fractures, specifically their waveguide-like capability of transporting energy (similar to electric current transported by metal wires). Hornby et al. (1989) and Kostek et al. (1998a) also show that the reflection amplitude of tube waves from a fracture is frequency dependent and increases as the frequency approaches zero.

There is strong evidence that an adequate theory of seismic wave propagation in fluidsaturated rock requires incorporating fracture fluid waves. From hydrogeology results, it follows that some scaling rules need to be applied to laboratory permeability measurements 
before using them at field scale. In hydrological experiments, the permeability parameter can be evaluated at the laboratory scale $(0.01-0.1 \mathrm{~m})$ by measuring fluid-flow rate through a rock sample in the presence of a fluid-pressure gradient. At the 10-100 m field scale, permeability is routinely evaluated using well tests (Muskat, 1946), and at a 100-1000 m scale, permeability is generally determined using tracer tests. Therefore, laboratory and field scales can differ by about 5 orders of magnitude. Measurements of permeability (hydraulic conductivity) for a wide range of scales have been done in a number of comprehensive studies for a variety of geologic environments (Clauser, 1992; Neuman, 1994; Shultze-Makuch et al., 1999; Shultze-Makuch and Cherkauer, 1998; and Gelhar, 1993). Typically, five-orders-ofscale increase corresponds to 5-7 orders of permeability increase. Such scaling behavior suggests the dominant role of fractures in fluid flow at field scales. Biot-Gassmann poroelasticity theory (Biot, 1962; 1956ab; Gassmann, 1951) treats fluids in fractures as flowing between rigid walls. It does not describe the existence of slow fluid waves, which require elastic walls. However, the importance of slow fluid waves for seismic wave propagation in fluid-bearing rock needs justification.

Rock fluids, such as water or oil, have some natural viscosity that causes attenuation of propagating fluid waves. At the same time, the thicknesses of real fractures might be on the order of millimeter or less. Can slow fluid waves propagate and cause resonances in real fractures? To answer this question, we need to evaluate for realistic parameters the attenuation of slow fluid waves caused by fluid viscosity, which is the subject of this paper.

\section{THEORY}




\section{Stoneley slow wave}

Consider a symmetric model of a layer $-h / 2 \leq z \leq h / 2$, filled with viscous fluid between two homogeneous elastic half-spaces (Figure 1) with the same material parameters. Here, I use derivations similar to those used by Ferazzini and Aki (1987). In the following, index $j=1$ indicates the parameters and fields related to the layer, while index $j=2$ indicates the values related to half-spaces. As also shown in Figure 1, I consider propagation of symmetrical wave modes along the OX axis of the $x$ spatial coordinate.

In a medium with non-zero shear modulus, two body waves can propagate: a longitudinal (P-) wave with velocity

$$
V_{P j}=\sqrt{\frac{\lambda_{j}+2 \mu_{j}}{\rho_{j}}}
$$

and a shear (S-) wave with velocity

$$
V_{S j}=\sqrt{\frac{\mu_{j}}{\rho_{j}}}
$$

expressed through Lame constants $\lambda_{j}, \mu_{j}$ and density $\rho_{j},(j=1,2)$.

Combining a linearized equation for compressible viscous fluid (Landau and Lifshitz, 1959) with a continuity relation, I obtain the equation for viscous-fluid-velocity motion, in the following form:

$$
\frac{\partial^{2} \mathbf{u}}{\partial t^{2}}-\frac{\eta}{\rho_{f}} \nabla^{2} \frac{\partial \mathbf{u}}{\partial t}-\frac{1}{\rho_{f}}\left(\xi-\frac{\eta}{3}\right) \nabla \nabla \square \frac{\partial \mathbf{u}}{\partial t}-c_{P}^{2} \nabla \nabla \square \mathbf{u}=0
$$

with time $t$, fluid density $\rho_{f}=\rho_{1}$, viscosity coefficients $\eta, \xi$, and speed $c_{p}$ of sound in the absence of viscosity. Particle velocity $\mathbf{u}$ in equation (4) can be represented as the sum

$$
\mathbf{u}=\mathbf{u}_{P}^{(1)}+\mathbf{u}_{S}^{(1)},
$$


where $\mathbf{u}_{P}^{(1)}$ and $\mathbf{u}_{S}^{(1)}$ are compressional and shear components, respectively. These components obey the equations

$$
\nabla \times \mathbf{u}_{P}^{(1)}=0, \quad \nabla \square \mathbf{u}_{S}^{(1)}=0,
$$

and satisfy equations of motion

$$
\frac{\partial^{2} \mathbf{u}_{P}^{(1)}}{\partial t^{2}}-\frac{1}{\rho_{f}}\left(\xi+\frac{4 \eta}{3}\right) \nabla \nabla \square \frac{\partial \mathbf{u}_{P}^{(1)}}{\partial t}-c_{P}^{2} \nabla \nabla \square \mathbf{u}_{P}^{(1)}=0
$$

and

$$
\frac{\partial^{2} \mathbf{u}_{S}^{(1)}}{\partial t^{2}}+\frac{\eta}{\rho_{f}} \nabla \times \nabla \times \frac{\partial \mathbf{u}_{S}^{(1)}}{\partial t}=0 .
$$

Using the time dependence of the fields in the form $\exp (-i \omega t)$, with angular frequency $\omega$, equations 7 and 8 correspondingly describe the propagation of dissipating P- and S- waves with complex velocities

$$
\begin{aligned}
& V_{\mathrm{P} 1}=\sqrt{c_{P}^{2}-i \frac{\omega}{\rho_{f}}\left(\xi+\frac{4 \eta}{3}\right)}, \\
& V_{S 1}=\sqrt{-\frac{i \omega \eta}{\rho_{f}}},
\end{aligned}
$$

and complex Lame constants

$$
\begin{aligned}
& \lambda_{1}=c_{P}^{2} \rho_{f}-i \omega\left(\xi-\frac{2 \eta}{3}\right), \\
& \mu_{1}=-i \omega \eta
\end{aligned}
$$

Shear waves in fluid appear exclusively because of viscosity and have diffusive propagation types. Displacements $\mathbf{u}_{p}^{(j)}$ and $\mathbf{u}_{s}^{(j)},(j=1,2)$ relate to potentials $\varphi_{j}$ and $\psi_{j}$ through the following equations: 


$$
\begin{aligned}
& \mathbf{u}_{P}^{(j)}=\nabla \varphi_{j}, \\
& \mathbf{u}_{S}^{(j)}=\nabla \times\left(\psi_{j} \mathbf{y}_{1}\right),
\end{aligned}
$$

where the unit vector $\mathbf{y}_{1}$ is used. I seek a solution in the form of a surface wave with wavenumber $k_{x}=\frac{\omega}{V_{f}}$, propagating along the $\mathrm{OX}$ axis with phase velocity $V_{f}$. For the symmetrical mode, the potentials have the forms

$$
\begin{aligned}
& \varphi_{1}=A_{1}\left(e^{k_{x} \sqrt{1-\alpha_{P 1}^{2}} z}+e^{-k_{x} \sqrt{1-\alpha_{P 1}^{2} z}}\right) e^{\mathrm{i} k_{x} x}, \\
& \psi_{1}=B_{1}\left(e^{k_{x} \sqrt{1-\alpha_{S 1}^{2} z}}-e^{-k_{x} \sqrt{1-\alpha_{S 1}^{2} z}}\right) e^{\mathrm{i} k_{x} x},
\end{aligned}
$$

inside the layer and

$$
\begin{aligned}
& \varphi_{2}=A_{2} e^{-k_{x} \operatorname{sign}(z) \sqrt{1-\alpha_{P 2}^{2} z}} e^{\mathrm{i} k_{x} x}, \\
& \psi_{2}=B_{2} e^{-k_{x} \operatorname{sign}(z) \sqrt{1-\alpha_{S 2}^{2} z}} e^{\mathrm{i} k_{x} x}
\end{aligned}
$$

outside the layer, where

$$
\alpha_{P j}=\frac{\omega}{\mathrm{k}_{\mathrm{x}} V_{P j}}=\frac{V_{f}}{V_{P j}}, \quad \alpha_{S j}=\frac{\omega}{k_{x} V_{S j}}=\frac{V_{f}}{V_{S j}}, \quad(j=1,2)
$$

and $A_{j}$ and $B_{j}$ are constants determined from the boundary conditions.

Boundary conditions at interfaces $z= \pm h / 2$ require continuity of displacements and stress, which have the components

$$
\begin{aligned}
& \tau_{x z}^{(j)}=2 \mu_{j} \frac{\partial^{2} \varphi_{j}}{\partial x \partial z}+\mu_{j}\left(\frac{\partial^{2} \psi_{j}}{\partial x^{2}}-\frac{\partial^{2} \psi_{j}}{\partial z^{2}}\right), \\
& \tau_{z z}^{(j)}=\lambda_{j} \nabla^{2} \varphi_{j}+2 \mu_{j}\left(\frac{\partial^{2} \psi_{j}}{\partial z^{2}}-\frac{\partial^{2} \psi_{j}}{\partial x \partial z}\right), \quad(j=1,2) .
\end{aligned}
$$


Since symmetry is embedded in the solution, we need to satisfy boundary conditions on just one interface. The problem has four unknowns $A_{j}, B_{j}(j=1,2)$, with four equations at the boundary using two components for both stress and displacement. I obtain the dispersion equation for symmetric modes by finding values of $\mathrm{V}_{f}$ for which the determinant of the system is zero. Using $z=h / 2$, we can obtain (after some algebra) the equation

$$
\begin{array}{r}
(1-c)^{2} \xi_{1} \xi_{2} \chi_{1} \chi_{2}-(b-c)^{2} \xi_{1} \chi_{1}-c(1-a)(1-b)\left[\xi_{2} \chi_{1}+\xi_{1} \chi_{2}\right]- \\
(1-a c)^{2} \xi_{2} \chi_{2}+(c a-b)^{2}=0,
\end{array}
$$

where

$$
\begin{aligned}
& \xi_{1}=\sqrt{1-\alpha_{P 1}^{2}} \tanh \left(\sqrt{1-\alpha_{P 1}^{2}} \frac{k_{x} h}{2}\right), \\
& \chi_{1}=\sqrt{1-\alpha_{S 1}^{2}} \operatorname{coth}\left(\sqrt{1-\alpha_{S 1}^{2}} \frac{k_{x} h}{2}\right), \\
& \xi_{2}=\sqrt{1-\alpha_{P 2}^{2}}, \\
& \chi_{2}=\sqrt{1-\alpha_{S 2}^{2}},
\end{aligned}
$$

and coefficients $a, b$, and $c$ have expressions

$$
a=1-\frac{\alpha_{S 1}^{2}}{2}, \quad b=1-\frac{\alpha_{S 2}^{2}}{2}, c=\frac{\mu_{1}}{\mu_{2}} .
$$

The roots of equation 22 provide all possible symmetrical modes for the problem. However, I am interested in only the low-frequency mode corresponding to the slow fluid wave. Assuming that both frequency $\omega$ and thickness $h$ are small enough, the argument in equation (23)

$$
\left|\sqrt{1-\alpha_{P 1}^{2}} \frac{k_{x} h}{2}\right|<<1 .
$$


In addition, if the velocity $V_{f}$ is small enough,

$$
\left|\alpha_{P 1}\right|<<1,\left|\alpha_{P 2}\right|<<1 \text { and }\left|\alpha_{S 2}\right|<<1
$$

and equation 22 yields

$$
\chi_{1}\left[\mathrm{k}_{x} h-\frac{\rho_{f} \alpha_{\mathrm{S} 2}^{2}}{\rho_{2}\left(1-\gamma^{2}\right)}\right]=2
$$

I also assume that phase velocity $V_{f}$ is much larger than shear wave velocity $V_{S 1}$ in the viscous fluid, providing the condition

$$
\left|\alpha_{S 1}\right|>>1
$$

For "thin" fractures, when

$$
\left|\sqrt{1-\alpha_{S_{1}}^{2}} \frac{k_{x} h}{2}\right| \approx \frac{S}{2}<<1,
$$

the hyperbolic function in equation 24 allows asymptotic expansion

$$
\chi_{1} \approx \frac{2}{k_{x} h}\left(1-\frac{k_{x}^{2} h^{2}}{12} \alpha_{S 1}^{2}\right)=\frac{2}{k_{x} h}(1+\beta)
$$

where parameter

$$
\beta=-i \frac{S^{2}}{12}
$$

is expressed through the normalized skin factor

$$
S=h \sqrt{\frac{\omega \rho_{f}}{\eta}} .
$$

From equation (30), I obtain

$$
V_{f}^{3}=V_{f 0}^{3} \frac{\beta}{1+\beta},
$$


in equation 36 , the parameter $V_{f 0}$ is given by equation 1 .

For $\beta<<1$, from equation 36 , I obtain

$$
V_{f}=h\left(-i \frac{\omega^{2} \mu_{2}}{12 \eta}\left(1-\gamma^{2}\right)\right)^{\frac{1}{3}}
$$

For " thick" fractures, when

$$
\left|\sqrt{1-\alpha_{s_{1}}^{2}} \frac{k_{x} h}{2}\right| \approx \frac{S}{2}>>1,
$$

and $\chi_{1} \approx-i \alpha_{S 1}$, equation 30 gives the solution

$$
V_{f}^{3}=V_{f 0}^{3}\left(1-\frac{1}{\sqrt{3 \beta}}\right)
$$

which approaches equation 1 of Ferrazzini and Aki (1987).

Equations 36 and 39 allow combining them into a general form

$$
V_{f}^{3}=V_{f 0}^{3} \frac{\beta}{1+\sqrt{\beta / 3}+\beta},
$$

where both asymtotics 32 and 33 are represented.

\section{Biot slow wave}

A fracture model with rigid walls (Figure 2) is also capable of carrying dispersive fluid waves with low velocity. In such a model, the interaction between walls and the fluid occurs through viscous friction forces. For thin fractures, the fluid motion is mostly directed along 
the walls with a parabolic distribution across the fracture, which reaches the maximum at the center line $\mathrm{z}=0$ and zero at the walls.

Introducing the total flow

$$
F=\frac{1}{h} \int_{0}^{h} \mathrm{u}_{\mathrm{x}} d z
$$

across any $x=$ const, reduces equation 4 to

$$
\frac{\partial^{2} F}{\partial t^{2}}+\frac{12 \eta}{h^{2} \rho_{f}} \frac{\partial F}{\partial t}-\frac{\xi+\frac{4 \eta}{3}}{\rho_{f}} \frac{\partial^{2}}{\partial x^{2}} \frac{\partial F}{\partial t}-c_{P}^{2} \frac{\partial^{2} F}{\partial x^{2}}=0
$$

Note that fracture permeability $\kappa_{f r}=h^{2} / 12$, therefore, it is embedded in the denominator of the second term of equation 42. In the frequency domain, equation 42 has the solution (Korneev et al, 2004)

$$
u=\exp (i \tilde{k} x) \exp (-i \omega t), \quad \tilde{k}=k+i \alpha
$$

with the wave number $k$, the attenuation coefficient $\alpha$ and the angular frequency $\omega$. The wavenumber components have the forms

$$
\begin{aligned}
& k=\frac{\omega}{c_{P}} \sqrt{\frac{\sqrt{(1+d g)^{2}+(d+g)^{2}}+1+d g}{2\left(1+g^{2}\right)}}, \\
& \alpha=\frac{\omega}{c_{P}} \sqrt{\frac{\sqrt{(1+d g)^{2}+(d+g)^{2}}-1-d g}{2\left(1+g^{2}\right)}},
\end{aligned}
$$

where

$$
d=\frac{12 \eta}{\omega h^{2} \rho_{f}}=\frac{\eta}{\omega \kappa_{f r} \rho_{f}}, \quad g=\frac{\omega\left(\xi+\frac{4 \eta}{3}\right)}{\rho_{f} c_{P}^{2}}
$$


At low frequencies, the phase velocity $V_{\mathrm{f}}$ of the wave described by equation 43 has the asymptotic form

$$
V_{\mathrm{f}}=V_{\mathrm{f}}^{\text {Biot }}=c_{P} \sqrt{\frac{2 \omega \kappa_{f r} \rho_{f}}{\eta}},
$$

similar to that for the slow Biot's wave (Biot, 1956a, equation 7.34). In fact, Biot uses the same model with rigid walls to describe the interaction of viscous fluid with rock pore walls (Biot, 1956b, page 180). Thus, the solutions 43-47 of equation 42 are the Biot-type solutions, in which the dispersive character of waves exists because of the finite viscosity of the fluid. At the zero viscosity, both parameters $d$ and $g$ in equations 46 vanish, and the wave given by equation 42 loses its frequency dependence. However, when the fracture-wall elasticity is taken into account, the existence of the slow fluid wave does not require a non-zero viscosity (equation 40). The asymptotics of Biot's slow wave can also be obtained from equation 22 (B. Gurevich, personal communication, 2007) as another symmetric mode. However, the author prefers solution 43-45 because of its explicit form in the entire frequency range.

\section{NUMERICAL RESULTS}

To estimate the possible effects of different fluids on the velocity and attenuation of the Stoneley guided wave, I perform computations for two parameter cases: a $1 \mathrm{~mm}$-thick fracture filled with water and oil that have the viscosities of 0.01 and 0.05 poises, respectively.

I compare five different solutions including the Biot's solution (equations 43-46), the nonviscous fluid solution by Ferrazzini and Aki, (equation 1), the exact solution for a viscous fluid, the general low-frequency asymptotic 40, and its forms 37 and 39. I obtain the exact solution for the viscous fluid by grid search for a complex root of equation 22 . The results are 
shown in Figures 3 and 4. For the entire frequency range $(1-1000 \mathrm{~Hz})$, the left-hand side of inequality 28 does not exceed $5 \times 10^{-3}$, while the right-hand side of inequality 31 is larger than $10^{4}$.

\section{DISCUSSION}

For both examined fluids (water and oil), Biot's solution substantially overestimates the Stoneley wave propagation velocities. This is predictable because the Biot's solution describes propagation of the body rather than surfacewaves. For water, the nonviscousfluid solution gives a good approximation for the entire range of frequencies, while for oil, it largely overestimates the velocities at low frequencies, where the differences between different fluids are most profound. The "thin" fracture asymptotic (equation 36) quite accurately predicts velocities at low frequencies, while yielding just a constant-limit value for the $Q$ factor. The general asymptotic (equation 40) reasonably describes the exact solution at all frequencies. Surprisingly, the low velocities of Stoneley waves at seismic frequencies, which are in the range of tens meters/second, result in the wavelengths of about 3 to $10 \mathrm{~m}$. Such wavelengths are comparable with the fracture lengths, and therefore the resonance conditions might be satisfied in finite fractures. Indeed, let us consider a horizontal fracture with the length $l$. Assuming the zero displacement condition $\mathrm{u}(0)=0$ at $x=0$ and "driving" displacement $\mathrm{u}(1)=\mathrm{u}_{0} \exp (-i \omega t)$ at $x=l$, we have the solution

$$
u=u_{0} A(\omega) \exp (-i \omega t), \quad A(\omega, x)=\frac{\exp \left(i \omega x / V_{\mathrm{f}}\right)-\exp \left(-i \omega x / V_{\mathrm{f}}\right)}{\exp \left(i \omega l / V_{\mathrm{f}}\right)-\exp \left(-i \omega l / V_{\mathrm{f}}\right)}
$$


where the phase velocity $V_{\mathrm{f}}$ is taken from equation 36. A numerical example, exhibiting resonant behavior of a slow fluid wave in a $4 \mathrm{~m}$ long fracture is shown in Figure 5 for waterand in Figure 6 for oil infill of a fracture with $1 \mathrm{~mm}$ thickness. In these figures, the amplitudesquare average

$$
E(\omega)=\frac{1}{l} \int_{0}^{l}\|A(\omega, x)\|^{2} d x
$$

is shown. The first resonant peak occurs at $14 \mathrm{~Hz}$ for water and at $12 \mathrm{~Hz}$ for oil. Predictably, the peaks for the water-filled fracture are sharper and higher than those for the oil-filled ones. After applying equation 1 for phase velocity in "thick" fractures, and using solution 48, the resonant frequencies can be estimated from the following formula:

$$
\omega_{k}=(1+2 k) \sqrt{\frac{\pi^{3} h \mu}{l^{3} \rho}\left(1-\gamma^{2}\right)}, \quad k=0,1,2, \ldots
$$

For $k=0$, equation 50 evaluates the first resonance frequency being equal to $13.8 \mathrm{~Hz}$, which is a good approximation for the exact resonant frequency. For a "thin" fracture, equation 37 can be applied, and the amplitude-square average (equation 49) has the form

$$
E(\omega)=\frac{1-\exp (-2 w)}{w}
$$

where

$$
w=\frac{l}{h}\left(\frac{12 \omega \eta}{\mu_{2}\left(1-\gamma^{2}\right)}\right)^{\frac{1}{3}}
$$

Figure 7 shows a comparison between the exact solution and approximation 51 for an oilfilled fracture with $0.1 \mathrm{~mm}$ thickness. Both exhibit an increase in amplitude response as the frequency approaches zero. This interesting behavior, as well as the velocity differences for water and oil infills (Figures 3 and 4), might be parts of mechanisms responsible for yet 
unexplained low-frequency signatures observed for hydrocarbon reservoirs (Goloshubin et. al., 2006; Castagna et. al., 2003),

Thus, the solution for the Stoneley slow waves generated in a viscous fluid indicates that even for a rather thin fracture, the quality factor $Q$ can be large enough to allow the fluid-wave resonances. Considering the statistical distribution of fracture sizes, we might expect that at any given frequency, resonant conditions will be satisfied for some fracture population. Then, it would be natural to expect a strong interaction of slow waves in systems of intersecting fluid-filled fractures.

The fluid-filled fractures represent strong waveguides with relatively large wave amplitudes. A qualitative estimate of an amplitude $A_{f}$ for a wave propagating with velocity $V_{f}$, in a medium with density $\rho_{f}$ compared to an incident wave with amplitude $A_{o}$ in a medium with parameters $V_{0}$ and $\rho_{0}$ has the approximate form

$$
\frac{A_{f}}{A_{0}}=\sqrt{\frac{V_{0} \rho_{0}}{V_{f} \rho_{f}}} .
$$

Using the values $V_{0}=4000 \mathrm{~m} / \mathrm{s}$ and $\rho_{0}=2.7 \mathrm{~g} / \mathrm{cm}^{3}$ for the incident p-wave, and $V_{f}=20 \mathrm{~m} / \mathrm{s}$ and $\rho_{f}=1 \mathrm{~g} / \mathrm{cm}^{3}$ for the Stoneley guided wave, we get a 25 -fold increase in the fluid-wave amplitude. Note that this estimate is consistent with the numerical results of Groenenboom and Falk (2000). Furthermore, at resonances, the amplitude rise is approximately equal to $Q$, and we might have an extra 10-fold amplitude increase (Figure 3).

Fractures play a major role in determining the permeability and direction of fluid flow in rocks at field scales. This is especially true for oil reservoirs. In most rocks and at all scales, fractures generally have a hierarchical self-similar distribution. Fluid-filled fractures provide sharp velocity contrasts with dispersive Stoneley fluid waves and are likely to be capable of strongly absorbing energy from passing seismic waves. The energy absorption can increase 
tenfold when the resonant conditions are met, which might create favorable conditions for nonlinear fluid-flow effects. The resonant conditions for fracture-fluid waves can exist even below typical seismic frequencies when the slow-wave velocities approach zero. Fracture systems also represent contrast scattering media for propagating seismic waves.

All the above phenomena should lead to strongly frequency-dependent propagation effects for seismic waves, which affect both velocity and attenuation. Existing poroelastic theories that consider fractures in their models (Barenblatt, 1960; Pride and Berryman, 2003ab; Mavko and Nur, 1975; Dvorkin,et al., 1994 ) treat fractures just as channels for viscous fluid flow similar to Biot's model and without incorporating physics for the Stoneley slow waves. It might be possible that such incorporation is critically important for wave-propagation effects in fractured reservoirs. A number of studies describe the elastic properties of materials containing fluid-saturated noninteracting fractures (Hudson, 1980, 1981; O'Connell, and Budiansky, B., 1977, Gabrin and Knopoff, 1978). It remains unclear whether the Stoneley slow wave survives in asymptotic solutions for small fractures. A careful numerical modeling of interaction between propagating body waves and fluid-filled fractures is needed to address these problems. However, such modeling presents a major computational challenge because of the coexistence of wavelengths that have dramatically different sizes.

An adequate theory that can be applied at field scales needs to incorporate fluid-elastic interactions in fractures and self-similar fracture distribution. For proper extraction of permeability information, the scattering of seismic waves caused by fracturing within such heterogeneous systems needs to be taken into account.

It is yet unclear how well the idealized fracture models used in this study represent fractures in real rocks that have complex surface topography and varying thicknesses. One can 
speculate that the details of fracture geometry are not very important at low frequencies. This problem will be studied in a series of laboratory measurements, which are already under way.

\section{CONCLUSIONS}

The analytical solution has been obtained for the phase velocity of the Stoneley guided wave for an infinite fracture filled with a viscous fluid. The solution suggests a possibility of differentiating between oil and water at low frequencies. It also suggests that resonances at seismic frequencies are possible at the reservoir scale. The Stoneley-wave (slow-wave) effect, which is not a part of any existing poroelastic theory, needs to be taken into account at field scales.

\section{ACKNOWLEDGMENTS}

This work was supported by the National Energy Technology Laboratory, Office of Fossil Energy Sciences, of the U.S. Department of Energy under Contract \#DE-AC03-76SF00098. The author thanks Jim Berryman, Dmitry Silin and Michael Schoenberg for helpful comments. The paper was greatly improved from discussions with Boris Gurevich.

\section{REFERENCES}

Barenblatt, G. I., Zheltov, I..P., and I..N. Kochina, 1960, Basic concepts in the theory of seepage of homogeneous liquids in fissured rocks: Journal of Applied Mathematics, v. 24, $1286-1303$. 
Biot, M.A., 1962, Mechanics of deformation and acoustic propagation in porous media: Journal of Applied Physics, 33, 1482-1498.

Biot, M.A., 1956a, Theory of propagation of elastic waves in a fluid-saturated porous solid. . I. . Low-frequency range: Journal of the Acoustical Society of America, 28, 168--178.

Biot, M.A., 1956b, Theory of propagation of elastic waves in a fluid-saturated porous solid. . II. . Higher frequency range: Journal of the Acoustical Society of America, 28, 179--191. Castagna, J.P., Sun, S., and S.R. Wu., 2003, Instantaneous spectral analysis: detection of lowfrequency shadows associated with hydrocarbons: The Leading Edge, 22(3), 120--127.

Chouet, B., 1986, Dynamics of a fluid-driven crack in three dimensions by the finitedifference method: Journal of Geophysical Research., 91 (B14), 13967-13992.

Clauser, C., 1992, Permeability of crystalline rocks, Eos Trans. AGU, 73(21), 233.

Dvorkin, J., Nolen-Noeksema, R. and A. Nur, 1994, A squirt flow mechanisms: a macroscopic description: Geophysics, 59, 428-438..

Dvorkin, J., and A. Nur, 1993, Dynamic poroelasticity: a unified model with the squirt and Biot mechanisms, Geophysics, 58, 524-533.

Ferrazzini, V., and K. Aki,, 1987, Slow waves trapped in a fluid-filled infinite crack: Implications for volcanic tremor, Journal of Geophysical Research, 92, 9215-9223.

Garbin, H.D. and L.Knopoff, 1978, Elastic moduli of a medium with liquid-filled cracks, Quarterly of Applied Mathematics, October, 301-303.

Gassmann, F., 1951, Uber die Elastizitat poroser Medien, Vier. Natur. Gesellschaft Zurich, 96, 1-23.

Gelhar, L. W., 1993, Stochastic Subsurface Hydrology, Prentice-Hall, Old Tappan, N. J. 
Goloshubin, G.M., Korneev V. A., Silin, D. B., Vingalov V.S. and C. VanSchuyer, 2006, Reservoir imaging using low frequencies of seismic reflections.: The Leading Edge, 25 (5), $527-531$

Goloshubin G.M., Krauklis P.V., Molotkov L.A., Helle H.B., 1994, Slow wave phenomenon at seismic frequencies: $63^{\text {rd }}$ Annual SEG Meeting, . 809-811.

Goloshubin G.M., Krauklis P.V., Molotkov L.A., Helle H.B., 1993, Slow wave in oilsaturated layer:, EAEG $55^{\text {th }}$ Annual Meeting.

Groenenboom, J. and J. Falk, 2000, Scattering by hydraulic fractures: Finite-difference modeling and laboratory data: Geophysics, 65, 612-622.

Groenenboom, J., and D. B. van Dam, 2000, Monitoring hydraulic fracture growth: Laboratory experiments: Geophysics, 65 (2), 603-611.

Groenenboom, J., and J. T. Fokkema, 1998, Guided waves along hydraulic fractures,: $67^{\text {th }}$ Annual SEG Meeting

Gurevich, B, 2002, Effect of fluid viscosity on elastic wave attenuation in porous rocks: Geophysics, 67(1), 264-270, 10.1190/1.1451798

Hornby, B. E., Johnson, D. L.,Winkler, K.W., and R. A. Plumb, 1989, Fracture evaluation using reflected Stoneley-wave arrivals: Geophysics, 54, 1274-1288.

Hudson, J.A., 1980, Overall properties of a cracked solid,: Mathematical. Proceedings of. . Cambridge. . Philosophical . Society., 99, 371-384.

Hudson, J.A., 1981, Wave speeds and attenuation of elastic waves in material containing cracks: Geophysical Journal of Royal Astronomical. Society, 64, 133--150.

Korneev, V. A., Goloshubin, G. M., Daley, T.V. and D.B.Silin, 2004, Seismic low-frequency effects in monitoring of fluid-saturated reservoirs: Geophysics, 69,(2), 522-532. 
Kostek, S., Johnson, D. L., and C. J., Randall, 1998, The interaction of tube waves with borehole fractures. Part I: Numerical models: Geophysics, 63, 800-808.

Kostek, S., Johnson, D.L., Winkler, and B. E. Hornby, 1998, The interaction of tube waves with borehole fractures, Part II: Analytical models, Geophysics, 63, 809-815, Krauklis, P. V., 1962, About some low frequency oscillations of a liquid layer in elastic medium: PMM, V01.26, N6, 1111-1115 (in Russian).

Landau, L.D., and , E.M. Lifschitz, 1959, Fluid Mechanics: Reading, MA, Pergamon Press. Molotkov L. A. and A. V. Bakulin, 1998, The effective model of a stratified solid-fluid medium as a special case of the Biot model: Journal of Mathematical Sciences. 91, No. 2, 2812-2826.

Mavko, G., and A. Nur, 1975, Melt squirt in asthenosphere: Journal of Geophysical research, 80, 1444-1448.

Muskat, M., 1946, The Flow of Homogeneous Fluids through Porous Media: Ann Arbor, MI, J.W. Edwards, Inc.

Neuman, S. P., 1994, Generalized scaling of permeabilities: Validation and effect of support scale, Geophysical Research Letters, 21 (5), 349-352.

O'Connell, R.J., and B. Budiansky,.1977, Viscoelastic properties of fluid saturated cracked solids:: Journal of . Geophysical . Research, 82, 5719--5735.

Paillet, F.L. and J.E. White, 1982, Acoustic models of propagation in the borehole and their relationship to rock properties: Geophysics, 47, 1215-1228.

Pride, S.R., and J.G., Berryman, 2003a, Linear dynamics of double-porosity dual-permeability materials. . I. Governing equations and acoustic attenuation,: Physics Review E, . E 68, 036603 
Pride, S.R., and Berryman, J.G., 2003b, Linear dynamics of double-porosity dual-permeability materials. . II. . Fluid transport equations: Physics Review, E68,036604 Schoenberg, M.A., 1984, Wave Propagation in Alternating Solid and Fluid Layers: Wave Motion, 6, 3, 303-320.

Schoenberg, M.A., 1983, Wave Propagation in a Finely Laminated Periodic Elastoacoustic Medium: Applied Physics Letters, 42, 4, 350-352.

Schulze-Makuch, D., and D. S. Cherkauer, 1998, Variations in hydraulic conductivity with scale of measurements during aquifer tests in heterogeneous, porous carbonate rock Hydrogeology Journal, 6, 204-215.

Schulze-Makuch, D., D. A. Carlson, D. S. Cherkauer, and P. Malik, 1999, Scale dependency of hydraulic conductivity in heterogeneous media: Ground Water, 37, 904-919. 


\section{FIGURES}

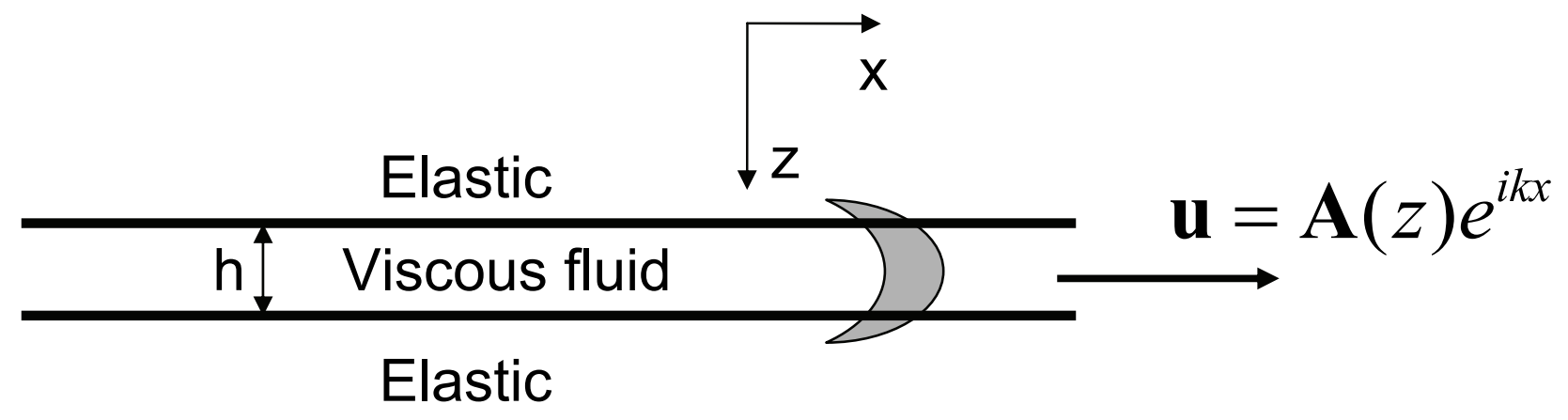

Figure 1. A layer filled with viscous fluid between two elastic halfspaces 


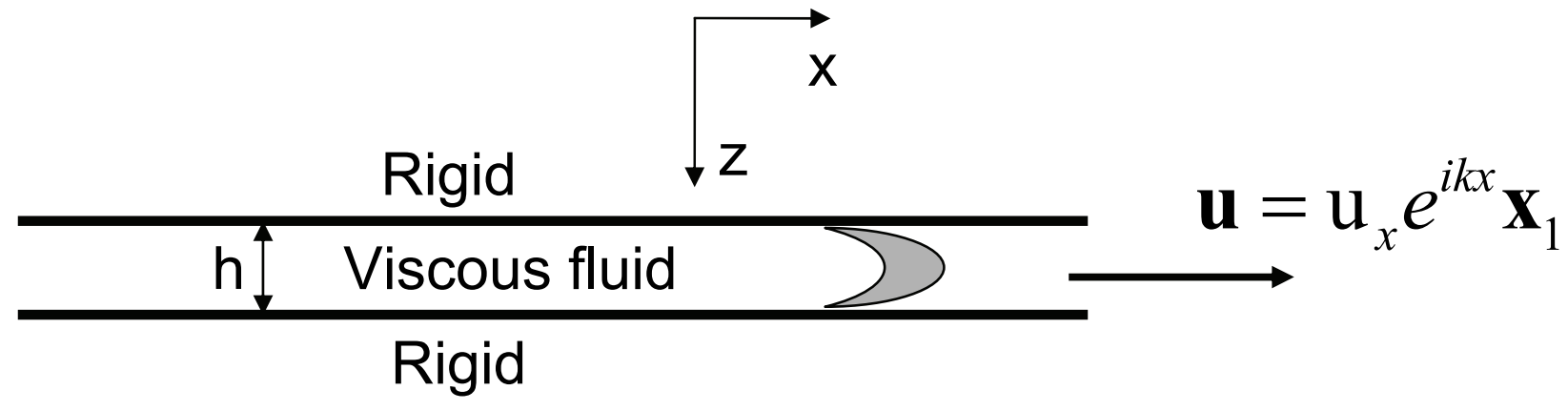

Figure 2. Biot's model for flow in fractures. Interaction of fluid with the fracture walls occurs because of the friction forces. Fluid motion goes predominantly along the rigid walls. 
(a)
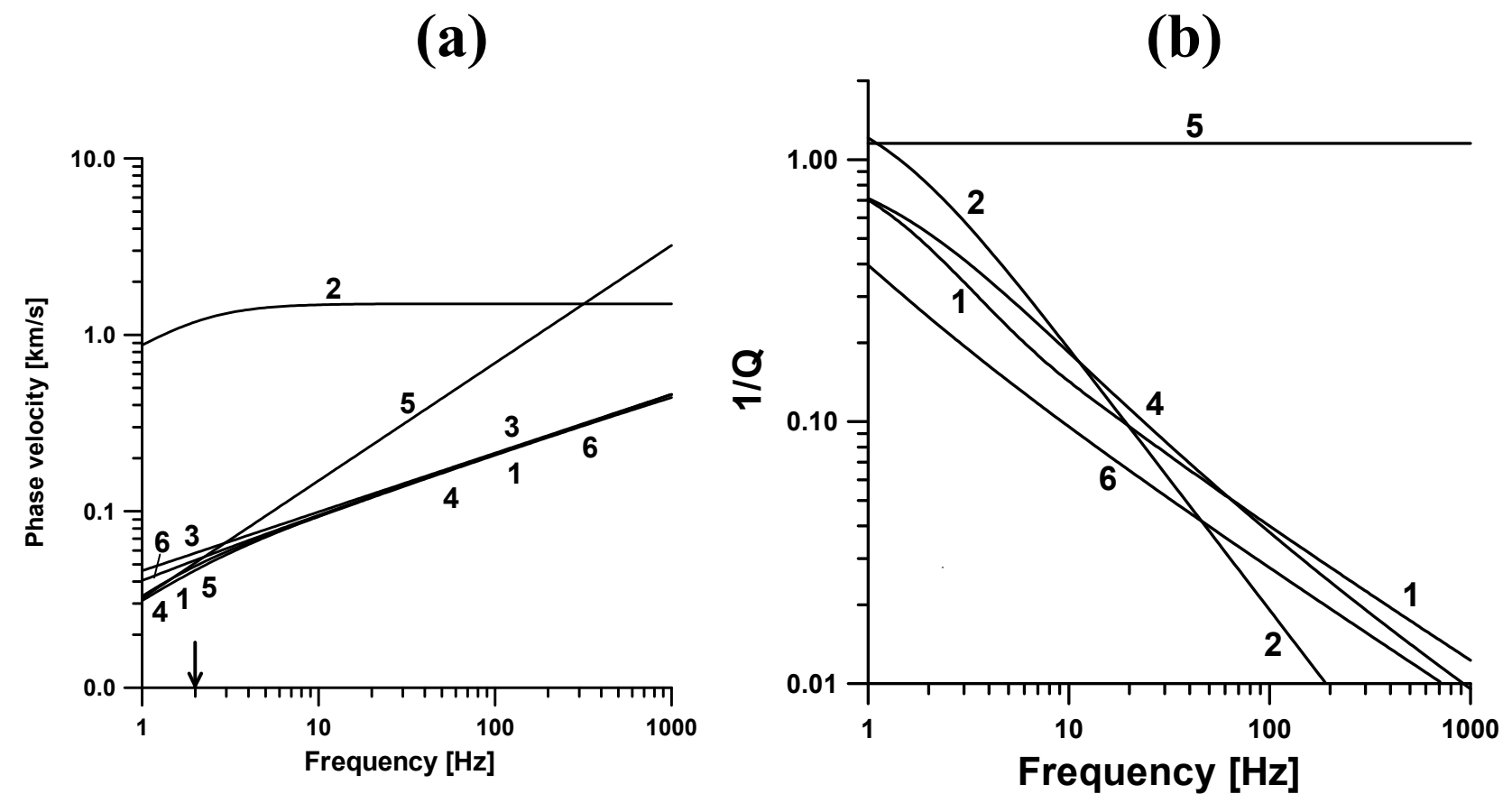

Figure 3. Comparison of solutions for a water-filled $1 \mathrm{~mm}$-thick fracture as functions of frequency. The curves are marked as follows: (1) exact solution for a viscous fluid in an elastic fracture computed via roots of equation 22; (2) Biot's solution from equations 43-45; (3) solution for nonviscous fluid given by equation 1; (4) general asymptotic (equation 40); (5) "thin" fracture asymptotic (equation 37), (6) "thick" fracture asymptotic (equation 39). (a) Phase velocities, (b) Inverse of attenuation factor Q. Note that the propagation velocities are small. While all fluid-elastic solutions have about the same velocity dependencies, the differences in attenuation are quite large. The values of $\mathrm{Q}$ at seismic frequencies reach 20. 
(a)

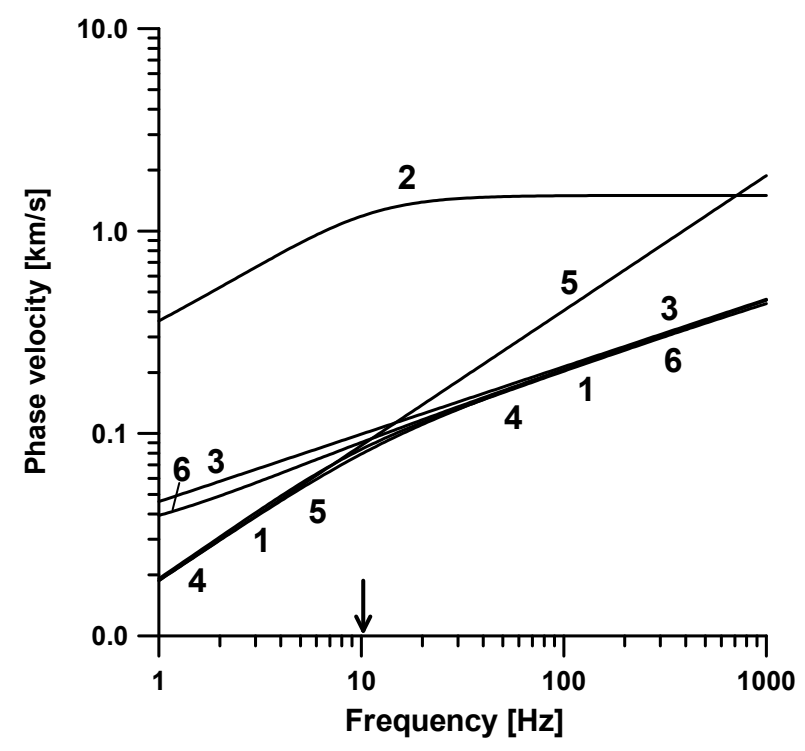

(b)

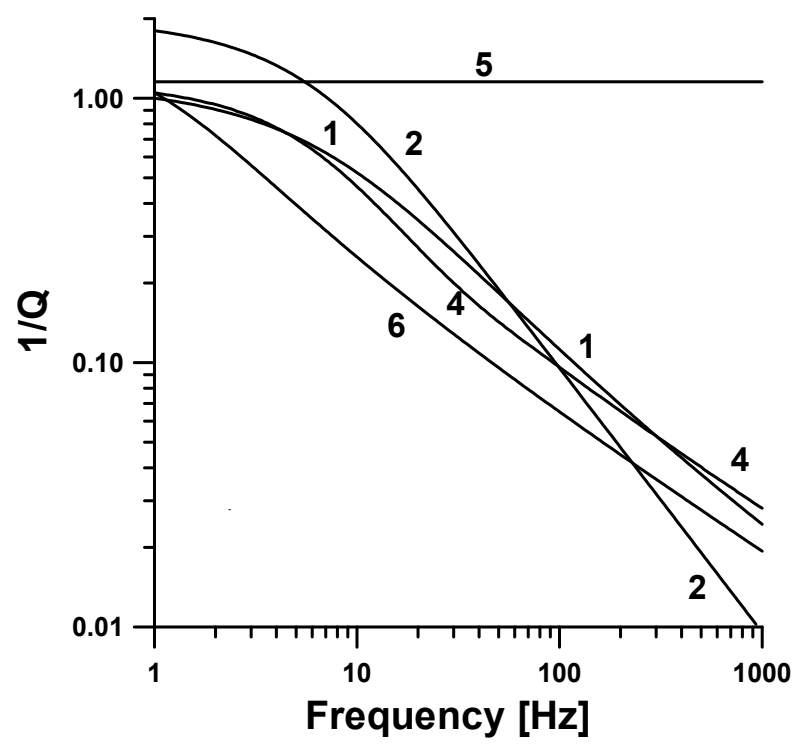

Figure 4. Same as in Figure 3, but for oil-filled fractures. The small $S$ asymptotic provides a good approximation for the exact solution, which differs significantly from the nonviscous fluid solution. The values of $\mathrm{Q}$ at seismic frequencies reach 10 . 


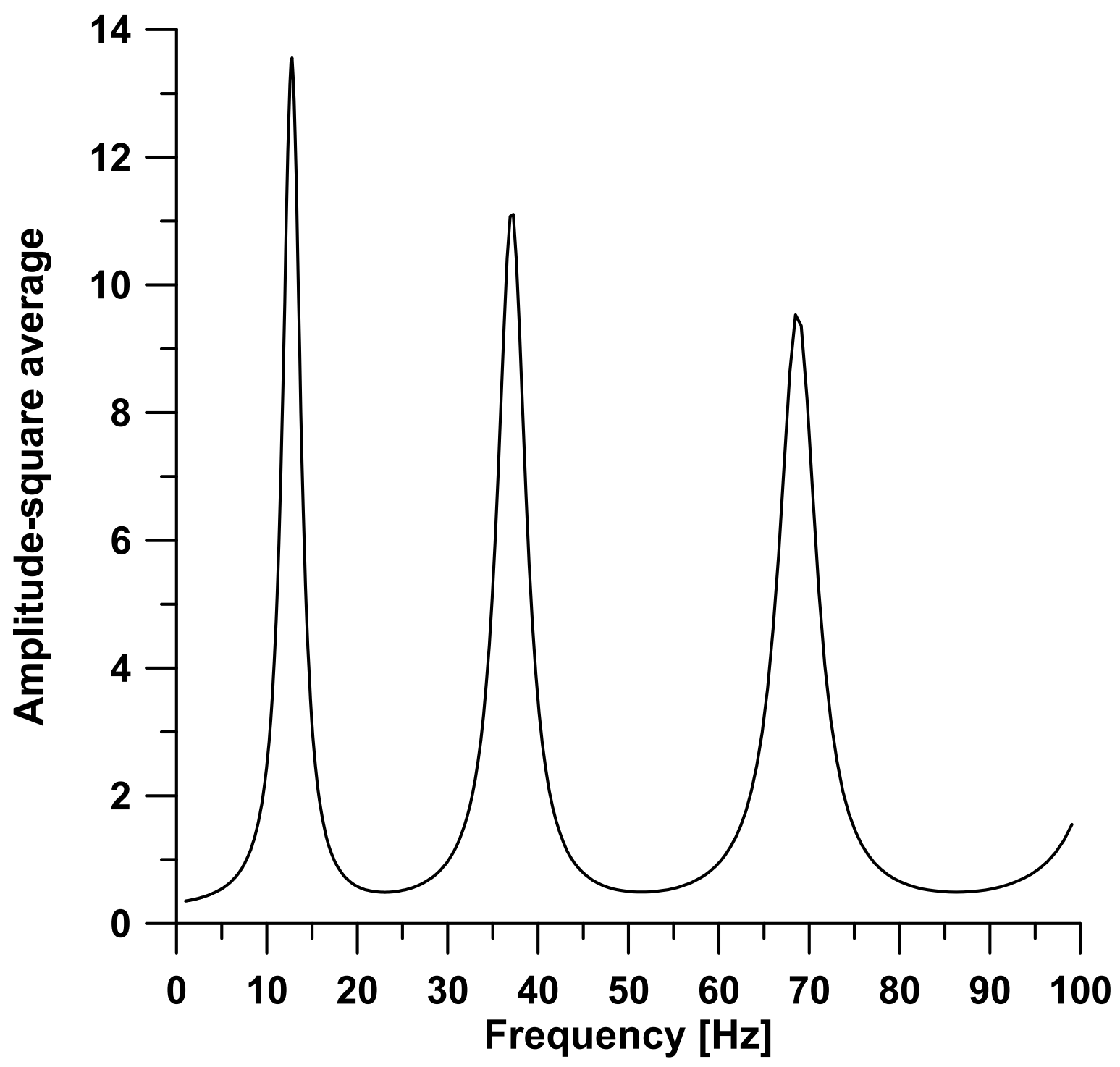

Figure 5. Resonant energy density for $4 \mathrm{~m}$ long $1 \mathrm{~mm}$ thick water-filled fracture. 


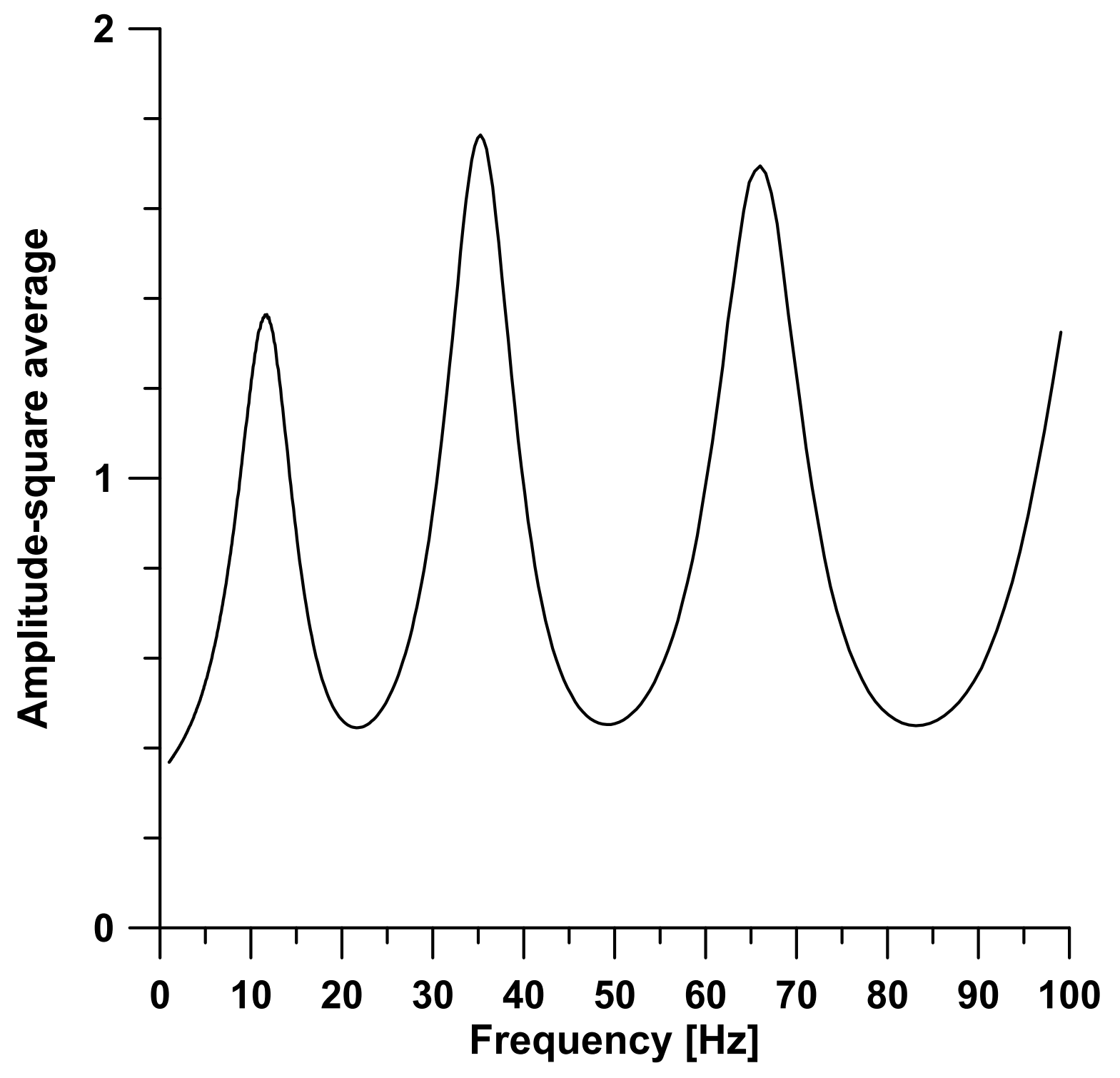

Figure 6. Same as in Figure 5, but for oil-filled fracture. 


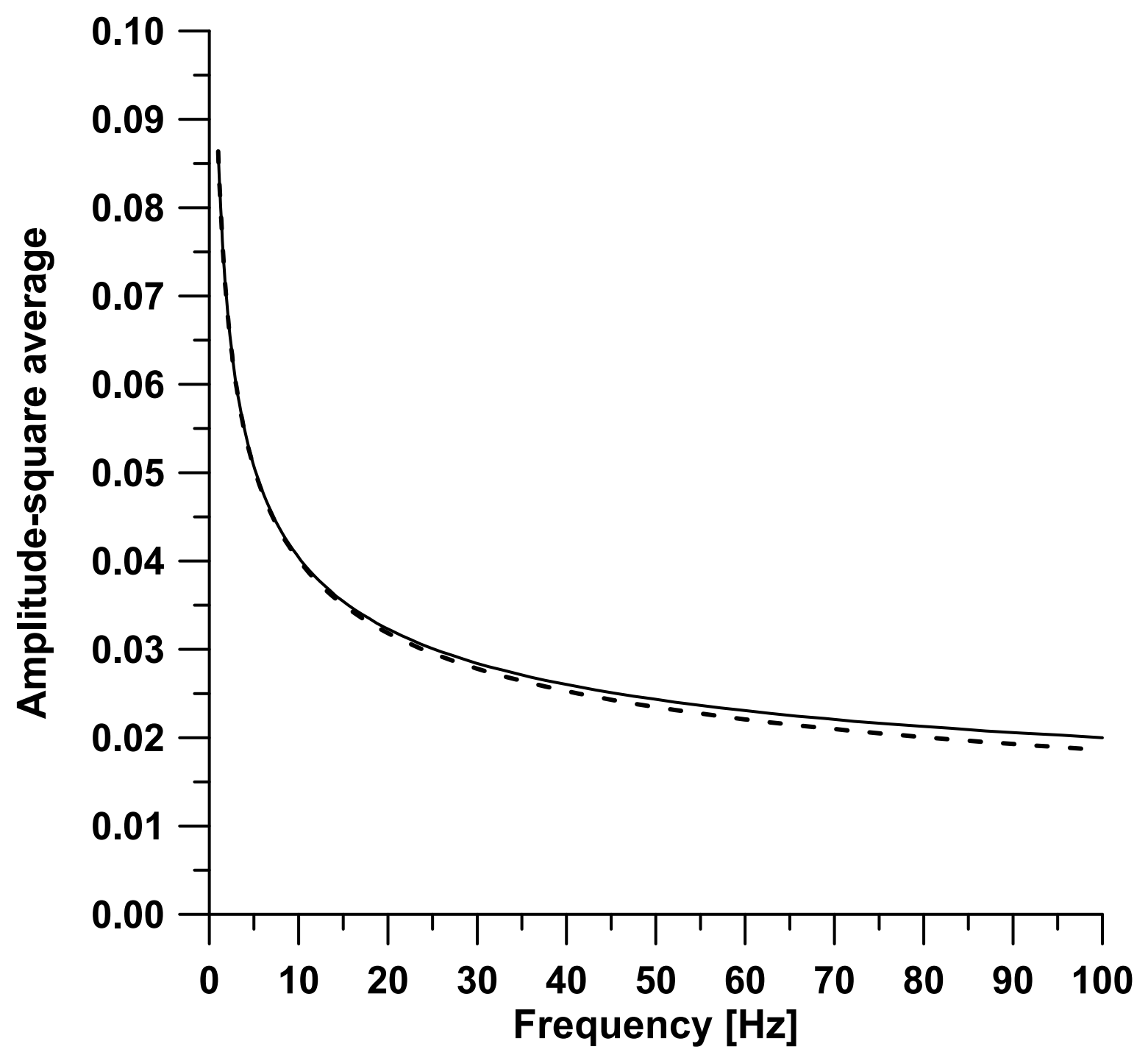

Figure 7. Amplitude-square average for a "thin" fracture filled with oil. Exact solution (solid line) is compared with analytical approximation given by equation 51 (dashed line). Low frequencies yeald higher amplitude responses. 


\section{Figure Captions}

\section{VG: Please correct in accordance with the captions below Figures.}

Figure 1. A layer filled with viscous fluid between two elastic halfspaces

Figure 2. Biot's model for flow in fractures. Interaction of fluid with the walls occurs because of friction forces. Fluid motion goes predominantly along the rigid walls.

Figure 3. Comparison of solutions for a water-filled $1 \mathrm{~mm}$ thick fracture as functions of frequency. The curves are marked as follows: (1) exact solution for a viscous fluid in an elastic fracture from roots of equation 22; (2) Biot's solution from equations 43-45; (3) solution for nonviscous fluid from equation 1; (4) general asymptotic from equation 40; (5) "thin" fracture asymptotic from equation 37, (6) "thick" fracture asymptotic from Equation 39. (a) Phase velocities, (b) Inverse of attenuation factor Q. Note the very small propagation velocities. Whereas all fluid-elastic solutions have about the same velocity dependencies, the difference in attenuation is quite large. The Q values reach 20 at seismic frequencies.

Figure 4. Same as in Figure 3, but now for oil-filled fractures. The small S asymptotic provides a good approximation for the exact solution, which differs significantly from the nonviscous fluid solution. Q values reach 10 at seismic frequencies.

Figure 5. Resonant energy density for $4 \mathrm{~m}$ long $1 \mathrm{~mm}$ thick water-filled fracture.

Figure 6. Same as in Figure 5, but for oil-filled fracture.

Figure 7. Amplitude-square average for "thin" fracture filled with oil. Exact solution (solid line) is compared with the analytical approximation from equation 51 (dashed line). Low frequencies give high amplitude response. 